\title{
Acute impairment of saccadic eye movements is associated with delayed cerebral ischemia after aneurysmal subarachnoid hemorrhage
}

\author{
*Matthew J. Rowland, DPhil, FRCA, ${ }^{1,2}$ Payashi Garry, FRCA, ${ }^{1,2}$ Jon Westbrook, FRCA, ${ }^{1,2}$ \\ Rufus Corkill, FRCR, ${ }^{2}$ Chrystalina A. Antoniades, PhD, ${ }^{1}$ and Kyle T. S. Pattinson, DPhil, FRCA ${ }^{1,2}$ \\ ${ }^{1}$ Nuffield Department of Clinical Neurosciences, University of Oxford; and ${ }^{2}$ Neurosciences Intensive Care Unit, Oxford University \\ Hospitals NHS Trust, Oxford, United Kingdom
}

\begin{abstract}
OBJECTIVE Delayed cerebral ischemia (DCl) causing cerebral infarction remains a significant cause of morbidity and mortality following aneurysmal subarachnoid hemorrhage (aSAH). Early brain injury in the first 72 hours following rupture is likely to play a key role in the pathophysiology underlying $\mathrm{DCl}$ but remains difficult to quantify objectively. Current diagnostic modalities are based on the concept of vasoconstriction causing cerebral ischemia and infarction and are either invasive or have a steep learning curve and user variability. The authors sought to determine whether saccadic eye movements are impaired following aSAH and whether this measurement in the acute period is associated with the likelihood of developing DCl.
\end{abstract}

METHODS As part of a prospective, observational cohort study, 24 male and female patients (mean age 53 years old, range 31-70 years old) were recruited. Inclusion criteria included presentation with World Federation of Neurosurgical Societies (WFNS) Grades 1 or 2 ("good grade") aSAH on admission and endovascular treatment within 72 hours of aneurysmal rupture. DCl and DCl-related cerebral infarction were defined according to consensus guidelines. Saccadometry data were collected at 3 time points in patients: in the first 72 hours, between Days 5 and 10, and at 3 months after aSAH. Data from 10 healthy controls was collected on 1 occasion for comparison.

RESULTS Age-adjusted saccadic latency in patients was significantly prolonged in the first 72 hours following aSAH when compared with controls (188.7 msec [ $95 \%$ Cl 176.9-202.2 msec] vs $160.7 \mathrm{msec}$ [95\% Cl 145.6-179.4 msec], respectively; $p=0.0054$, t-test). By 3 months after aSAH, there was no significant difference in median saccadic latency compared with controls (188.7 msec [95\% Cl 176.9-202.2 msec] vs $180.0 \mathrm{msec}$ [95\% Cl 165.1-197.8 msec], respectively; $p=0.4175$, t-test). Patients diagnosed with cerebral infarction due to $\mathrm{DCl}$ had a significantly higher age-adjusted saccadic latency in the first 72 hours than those without infarction $(240.6 \mathrm{msec}[95 \% \mathrm{Cl} 216.7-270.3 \mathrm{msec}]$ vs 204.1 $\mathrm{msec}[95 \% \mathrm{Cl} 190.7-219.5 \mathrm{msec}]$, respectively; $p=0.0157$, t-test). This difference was more pronounced during Days 5-10 following aSAH, the peak incidence for $\mathrm{DCl}(303.7 \mathrm{msec}[95 \% \mathrm{Cl} 266.7-352.7 \mathrm{msec}]$ vs $207.6 \mathrm{msec}[95 \% \mathrm{Cl}$ 193.7-223.6 msec], respectively; $p<0.0001$, t-test). A binary generalized linear model showed that latency in the first 72 hours was the only significant predictor of cerebral infarction $(p=0.0185)$.

CONCLUSIONS This is the first study to use saccadometry to measure the saccadic latency of eye movements in patients with aSAH during the acute period following aneurysm rupture. The results showed that median saccadic latency is associated with the risk of developing cerebral infarction due to $\mathrm{DCl}$ and may act as a potential objective biomarker to guide the need for intensive care admission and treatment. Future studies will look to formally validate saccadic latency as a biomarker of $\mathrm{DCl}$ in a larger cohort and assess whether the addition of saccades improves current clinical models for predicting patients at risk.

https://thejns.org/doi/abs/10.3171/2016.8.JNS16408

KEY WORDS brain injury; subarachnoid hemorrhage; saccadic eye movement; delayed cerebral ischemia; DCl; cerebral infarction; vascular disorders

\footnotetext{
ABBREVIATIONS aSAH = aneurysmal subarachnoid hemorrhage; $\mathrm{DCl}$ = delayed cerebral ischemia; $\mathrm{GCS}$ = Glasgow Coma Scale; $\mathrm{TCD}=$ transcranial Doppler; WFNS = World Federation of Neurosurgical Societies.

SUBMITTED February 18, 2016. ACCEPTED August 5, 2016.

INCLUDE WHEN CITING Published online December 9, 2016; DOI: 10.3171/2016.8.JNS16408.

* Drs. Antoniades and Pattinson share senior authorship of this work.
} 
$\mathrm{T}$ HE acute management of patients with brain injury following insults such as trauma, stroke, and cardiac arrest suffers greatly from the absence of an objective and noninvasive marker of tissue damage. Unlike the clinical assessment of patients with chest pain, who can be rapidly triaged into high and low risk based on an electrocardiogram and blood test, quantifying the severity of brain injury remains largely dependent on clinical history (e.g., mechanism of injury), subjective clinical assessment, and neuroimaging of anatomical structures. What is needed is a rapid and noninvasive measurement of neurological function that might allow clinicians to rapidly triage patients with acute brain injury into high and low risk categories, guide therapeutic outcomes, and prioritize intensive care admissions.

This is especially true for aneurysmal subarachnoid hemorrhage (aSAH). In those patients who survive the initial rupture to radiological or surgical aneurysm treatment, the most important cause of mortality and morbidity is a syndrome of neurological and cognitive deficits termed delayed cerebral ischemia (DCI). ${ }^{32}$ DCI affects up to a third of patients and is difficult to predict and diagnose and therefore often results in irreversible cerebral infarction and poor functional outcomes. ${ }^{8,15}$

Recently, the focus of research into the pathophysiology underlying DCI has moved from cerebral vasoconstriction to include damage to the brain in the first 72 hours following the bleed, so-called "early brain injury." This damage includes derangements of cerebral physiology that can lead to decreased cerebral blood flow, increased intracranial pressure, and neuronal cell death. ${ }^{6}$ Although these derangements are before the onset of DCI, it appears probable that the physiological changes occurring at this time may directly influence the likelihood and severity of later ischemic complications in patients after aSAH. This latent period between early brain injury and the development of DCI therefore offers the potential for individualized and "goal-directed" cerebral resuscitation to treat cerebral ischemia and prevent cerebral infarction if patients at risk can be identified.

Unfortunately, it remains difficult to objectively quantify early brain injury and diagnose DCI in patients following aSAH. The sensitivity and specificity of commonly used neurological scoring tools such as the Glasgow Coma Scale remain unknown and depend on a relatively large clinical neurological deterioration to diagnose DCI. Traditional imaging strategies following aSAH are aimed at the identification of cerebral vasoconstriction on the basis of the now erroneously presumed linear relationship between vessel narrowing, DCI, cerebral infarction, and poor neurocognitive outcome. ${ }^{25}$ Digital subtraction angiography is invasive, involves exposure to ionizing radiation, and has a low positive predictive value in diagnosing cerebral infarction after aSAH. It also correlates poorly with functional outcomes. ${ }^{10}$ Noncontrast CT is often requested to exclude rebleeding or hydrocephalus but has poor sensitivity to the detection of early cerebral ischemia and infarction. ${ }^{7}$ Finally, transcranial Doppler (TCD) ultrasonography is often used to screen for DCI by the detection of changes in large cerebral vessel flow velocities. Despite a large systematic review suggesting high sensitivity to the prediction of DCI, ${ }^{16}$ there remain significant barriers to the routine clinical use of TCD due to the fact that the technique has a prolonged learning curve, ${ }^{22}$ is highly operator dependent ${ }^{14,31}$ and $10 \%-20 \%$ of patients have temporal acoustic windows that do not allow reliable TCD measurement. ${ }^{20}$

Previous studies have shown that measurements of the time taken for the eyes to respond to a moving visual stimuli (saccadic latency) can be used reliably as an objective biomarker of neurocognitive function in more chronic conditions such as Huntington's and Parkinson's diseases. $^{3,4}$ Recently, prolonged saccadic latencies have also been reported in patients recovering from acute traumatic head injury. ${ }^{11,12,24}$ However, to date, no studies have investigated the utility of saccadometry in assessing neurological function in the acute period following brain injury.

Saccadic reaction times reflect the function of global brain circuitry involving the brainstem, cerebellum, basal ganglia, and cortex..$^{18,21}$ Lesion studies $^{30,33}$ and MRI evidence $^{27}$ indicate that the cerebellum is predominantly responsible for the adaptation of visually guided (reflexive) saccades. The adaptation of other types of saccades, however, may be mainly mediated by the frontal eye field (scanning saccades) or the dorsolateral prefrontal cortex (memory-guided saccades). ${ }^{5}$ In addition, there are suggestions that structures that are functionally further "downstream" in the initiation of saccades, such as the basal ganglia, are also important for these adaptation processes. ${ }^{13}$ Assessing the integrity of these brain structures and pathways through saccadometry is therefore likely to be of considerable interest in patients with other types of global brain injury such as that observed in aSAH, especially during the acute phase.

In this study, we aimed to investigate the efficacy of using a portable saccadometer acutely within the first 72 hours after aneurysmal rupture and at follow-up to measure eye movements in patients with aSAH. Given the likely poor clinical status of patients in the first hours following aneurysmal rupture, we focused on visually guided reflexive saccades to improve the accuracy of results and patient compliance. We hypothesized that these eye movements would provide a sensitive and objective functional marker of early brain injury in aSAH. In particular, we hypothesized that saccadic latency would be increased acutely following aneurysmal rupture and would correlate with the incidence of DCI/cerebral infarction after aSAH.

\section{Methods}

Ethics approval was granted by the Berkshire National Research Ethics Service Committee South Central as part of a larger research study of patients with World Federation of Neurosurgical Societies (WFNS) Grades 1 or 2 ("good grade") aSAH undertaken between March 2011 and December 2014. Male and female patients were eligible for inclusion if they were between 18 and 90 years of age and admitted within 72 hours of aneurysm rupture, and able to give consent to participating in the study. $\mathrm{Pa}-$ tients were recruited following coil embolization and written informed consent was obtained in all cases.

Ten healthy volunteers were also recruited as a control 
reference group, matched as a group by age to the patients recruited in the study (mean age 54 years, range 29-67 years). These control subjects were in good health with no significant medical history and no previous stroke or cerebral hemorrhage, and they were not taking any regular medications.

\section{Recording Saccadic Eye Movements}

Visually evoked horizontal saccades were recorded acutely at 3 time points: 1 ) Session $1,<72$ hours following aneurysmal rupture; 2) Session 2, 5-10 days after aSAH; and 3) Session 3, 3 months after aSAH.

Control subjects underwent 1 session of saccadometry recording only. A miniaturized infrared $1 \mathrm{kHz}$ saccadometer was used to measure saccadic latency (Fig. 1). The device was worn on the head, secured by an elastic strap, and resting on the bridge of the nose; 3 built-in low-power lasers projected red spots subtending some $0.1^{\circ}$ in a horizontal line in the midline at $\pm 10^{\circ} .1,23$ Participants were invited to lie flat and the red spots were projected onto the ceiling (white background). Because the stimuli move exactly with the head, no head restraint was necessary and sessions were well tolerated throughout the study, with no patients declining testing.

In each trial, the central fixation target was displayed for a random fore period of 1.0-2.0 seconds. The target then randomly moved to 1 of 2 peripheral positions on the left and right and remained illuminated until $200 \mathrm{msec}$ after the end of the saccade. ${ }^{3}$ Patients were instructed to keep their heads still and follow the target with their eyes as it moved. A single experimental run consisted of 12 calibration trials followed by 80 experimental trials and lasted approximately 3 minutes; aberrant records contaminated by excessive head movement and blinks were automatically removed by proprietary software. This software also determined the saccadic latency using a saccade-detection algorithm based on velocity and acceleration.

\section{Clinical and Neurocognitive Assessment}

DCI and cerebral infarction were defined as per consensus guidelines. ${ }^{32}$ Clinical deterioration due to DCI was defined as the occurrence of focal neurological impairment (such as hemiparesis, aphasia, apraxia, hemianopia, or neglect), or a decrease of at least 2 points on the Glasgow Coma Scale (GCS; either on the total score or on one of its individual components [eye, motor on either side, verbal]). This should last for at least 1 hour, is not apparent immediately after aneurysm occlusion, and cannot be attributed to other causes by means of clinical assessment, CT scanning or MRI of the brain, and appropriate laboratory studies. Cerebral infarction was defined as the presence of cerebral infarction on CT or MRI of the brain within 6 weeks after aSAH, or on the latest CT or MR image made before death within 6 weeks (or proven at autopsy), not present on the CT or MR scan between 24 and 48 hours after early aneurysm occlusion, and not attributable to other causes such as surgical clipping or endovascular treatment. Hypodensities on CT resulting from ventricular catheter or intraparenchymal hematoma were not regarded as cerebral infarctions from DCI.

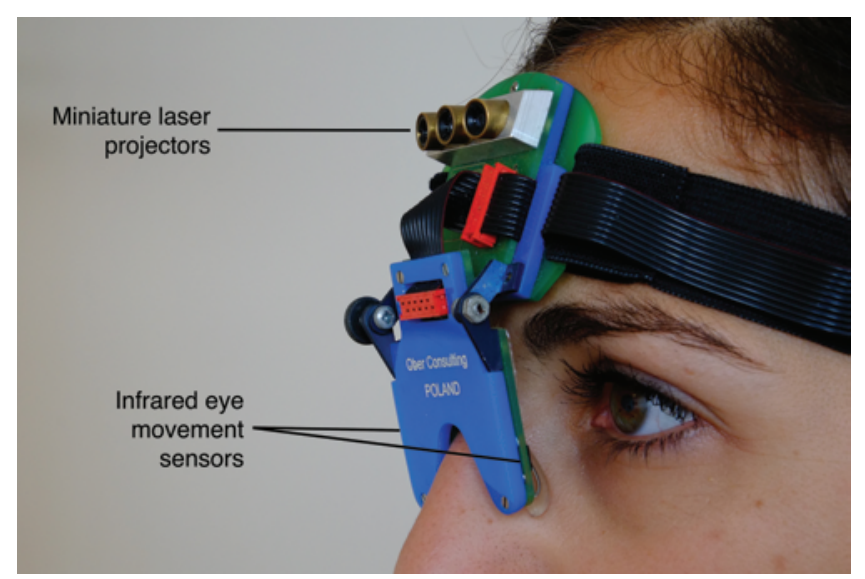

FIG. 1. Saccadometer headpiece (oculometer), worn across the bridge of the nose and against the forehead. The headpiece contains 3 lasers to project either a central fixation point or a visual target to the left or right of the central fixation, to which the participants were instructed to follow with their eyes.

All patients received clinical management as per the standard protocol at our institution, including nimodipine for 28 days following aSAH. Routine TCD or CT angiography was not performed because this is not standard practice at our institution. During the acute admission, a member of the research team who was blinded to the saccadometry results reviewed study participants daily. Changes in clinical condition were noted as well as the requirement for any new interventions, such as CSF drainage.

\section{Power Calculation and Statistical Analysis}

A formal power calculation was difficult to perform in this study because this is the first study to investigate saccadometry acutely in patients with aSAH. Based on the incidence rate of DCI of approximately $30 \%$ in patients, we estimated a sample size of 16 patients to detect a frequency of this event with $80 \%$ power.

Preprocessing of saccadometry data was performed by a member of the research team (C.A.A.) blinded to clinical outcome using custom software (LatencyMeter, Ober Consulting). Statistical analysis was performed using Prism 6 (GraphPad Software) and R software. A 2-level multilevel model was fitted to account for age and the repeated measurements of saccadic latency taken at 3 time points on each patient; normality and homoscedasticity were found to be well satisfied by taking the reciprocal of latency as the response variable (Shapiro-Wilk test, $p$ $=0.3927$ ). Two further multivariate analyses were used to investigate the effects of saccadic latency on cerebral infarction, including potential confounding factors as covariates (age, sex, WNFS grade, and Fisher grade). A binary generalized linear model was fitted successfully using the reciprocal of baseline latency as a predictor. To account for latency as a random variable that was subject to measurement variability, the binary regression model was refitted using a Bayesian approach. Further details regarding the statistical methods used can be found in the Supplementary Information. 
TABLE 1. Demographics of the study population

\begin{tabular}{lll}
\hline \multicolumn{1}{c}{ Variable } & No DCl & $\mathrm{DCl}$ \\
\hline No. of patients & 14 & 8 \\
\hline Mean age in yrs (range) & $51.64(31-70)$ & $56.25(39-69)$ \\
\hline Men/women & $6 / 8$ & $2 / 6$ \\
\hline WFNS grade & & 5 \\
\hline 1 & 10 & 3 \\
\hline 2 & 4 & \\
\hline Fisher grade (\%) & & 0 \\
\hline 1 & 0 & 1 \\
\hline 2 & 0 & 4 \\
\hline 3 & 1 & 3 \\
\hline 4 & 13 & \\
\hline Location of aneurysm & & 4 \\
\hline Anterior circulation & 3 & 2 \\
\hline Middle cerebral artery & 4 & 0 \\
\hline Internal carotid artery & 6 & 2 \\
\hline Posterior circulation & 1 & 5 \\
\hline Lt side & 9 & 1 \\
\hline Rt side & 5 & \\
\hline Midline & 0 & \\
\hline Hydrocephalus & 9 & \\
\hline None & 4 & \\
\hline Mild/moderate & & \\
\hline Severe & & \\
\hline
\end{tabular}

\section{Results}

Twenty-four patients were recruited to the study in total and underwent measurement of saccadic latency acutely (i.e., within 72 hours of aneurysm rupture). Two patients were subsequently excluded from the study due to significant cranial nerve palsy affecting eye movements and preventing measurement of saccades. Of the 22 patients who underwent successful acute saccadometry, 20 returned for saccades follow-up at 3 months after aSAH. In total, 8 patients were diagnosed with cerebral infarction as per the consensus guidelines above. Four of these patients were diagnosed with clinical deterioration due to DCI during the acute hospital admission. A further 4 patients were not diagnosed as having clinical symptoms of DCI during the admission but had new evidence of DCI-related cerebral infarction as per consensus guidelines on follow-up MRI. Demographics for all 22 patients studied are shown in Table 1.

Age-adjusted saccadic latency in patients was significantly prolonged in the first 72 hours following aSAH when compared with controls (188.7 msec [95\% CI 176.9$202.2 \mathrm{msec}$ ] vs $160.7 \mathrm{msec}$ [95\% CI 145.6-179.4 msec], respectively; $\mathrm{p}=0.0054$, t-test; Fig. 2). By 3 months after aSAH, there was no significant difference in median saccadic latency compared with controls (188.7 msec [95\% CI 176.9-202.2 msec] vs $180.0 \mathrm{msec}$ [95\% CI 165.1-197.8 msec], respectively; $\mathrm{p}=0.4175$, t-test). Patients diagnosed with cerebral infarction due to DCI had a significantly higher age-adjusted saccadic latency in the first 72 hours than those without infarction (240.6 msec [95\% CI 216.7$270.3 \mathrm{msec}$ ] vs $204.1 \mathrm{msec}$ [95\% CI 190.7-219.5 msec], respectively; $p=0.0157$, t-test; Fig. 3). This difference was more pronounced during Days 5-10 after aSAH, the peak incidence for DCI (303.7 $\mathrm{msec}$ [95\% CI 266.7-352.7 $\mathrm{msec}$ ] vs 207.6 [95\% CI 193.7-223.6 msec], respectively; $\mathrm{p}<$ 0.0001 , t-test). A binary generalized linear model showed that latency in the first 72 hours was the only significant predictor of cerebral infarction $(\mathrm{p}=0.0185)$. The addition of age, sex, and WFNS grade as predictors did not significantly improve the model $(\mathrm{p}=0.9465)$; there was weak evidence of an improvement from including observation of Fisher grade but it was not significant $(\mathrm{p}=0.0858$; see Supplementary Material for further details on statistical methods used).

Assuming a cutoff value of $215 \mathrm{msec}$ for median saccadic latency in the first 72 hours following rupture in our study population (Fig. 3, dashed line) gives a sensitivity of $88 \%$, a specificity of $65 \%$, and positive predictive value of 0.70 for detecting patients progressing to DCI and cerebral infarction (Supplementary Material, Fig. A).

There were no significant differences in age $(\mathrm{p}=$ $0.3734)$, sex $(p=0.6494)$, or WFNS grade $(p>0.99)$ between those patients with DCI and those without (Table 1). Interestingly, in this study modified Fisher grade-often used as a risk factor for DCI-on admitting CT was significantly higher in those patients without cerebral infarction at 6 months $(\mathrm{p}=0.0091)$. Only 1 patient required insertion of a ventriculoperitoneal shunt and this was in the noninfarction group on Day 18 after aSAH.

\section{Discussion}

In this study, we have prospectively investigated the utility of acutely measuring eye movement in critically unwell patients with aSAH and correlated these changes with short-term outcomes. There are a number of notable results. First, our results demonstrate that reflexive saccadic eye movement is adversely affected in patients in the first 72 hours following aneurysm rupture and remains impaired on Days 5-10 when compared with healthy controls. By 3 months after aSAH, median saccadic latency returns to healthy control levels. Most interestingly, those patients who developed DCI had significantly slower saccades in the first 72 hours and between Days 5 to 10 (the peak incidence for DCI) than those patients unaffected by DCI. Finally, the use of saccadometry acutely following aSAH is able to predict patients at risk for DCI with $88 \%$ sensitivity, equivalent to other current imaging modalities and clinical models in use after aSAH, and it is a noninvasive modality.

There is now evidence that factors relating to the initial aneurysm rupture such as clinical grades of severity (e.g., WFNS and Hunt and Hess grades) and the thickness of subarachnoid blood (as measured by Fisher score) may not correlate well with functional and neurocognitive outcomes. ${ }^{19}$ It is therefore likely that secondary ischemic damage occurring in the first hours following aneurysm rupture due to early brain injury may play a much more important role in predicting subsequent recovery following aSAH. ${ }^{28,29}$ As a result, there has been increasing inter- 


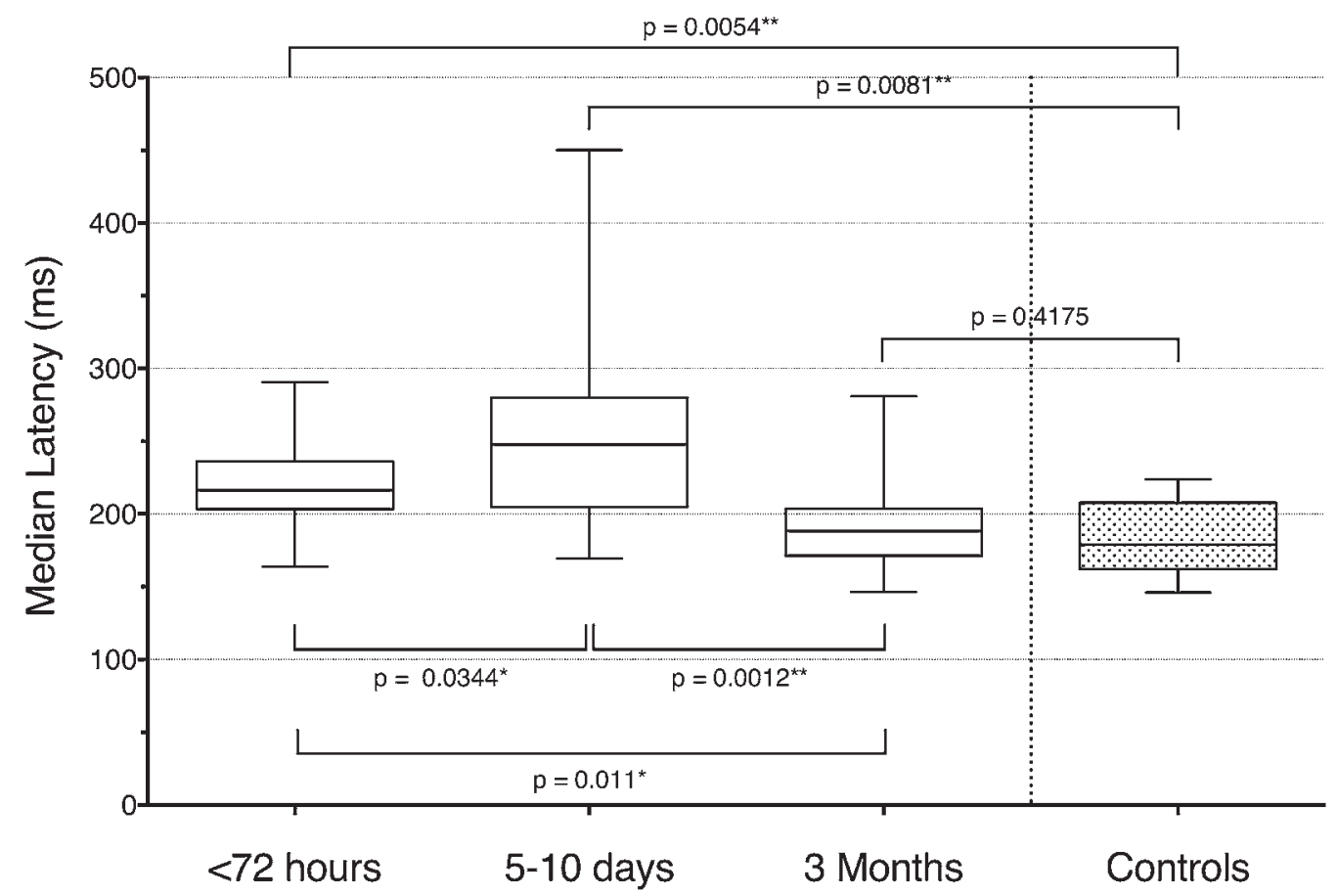

FIG. 2. Comparisons of median saccadic latency between patients and healthy controls (top bars with $p$ values) and longitudinal differences in median saccadic latency for patients in the first 72 hours after aSAH, Days 5-10, and 3 months after aSAH (bottom bars with $p$ values). ${ }^{*} p<0.05,{ }^{* *} p<0.01$.

est in the use of multimodal monitoring following aSAH to optimize the diagnosis and individualize therapeutic interventions aimed at preventing secondary neuronal injury. ${ }^{26}$ However, the majority of techniques currently in use to objectively quantify DCI are either unsuitable for monitoring those patients with "good grade" aSAH who do not require sedation/mechanical ventilation (e.g., tissue oxygen and intracranial pressure monitoring), or have

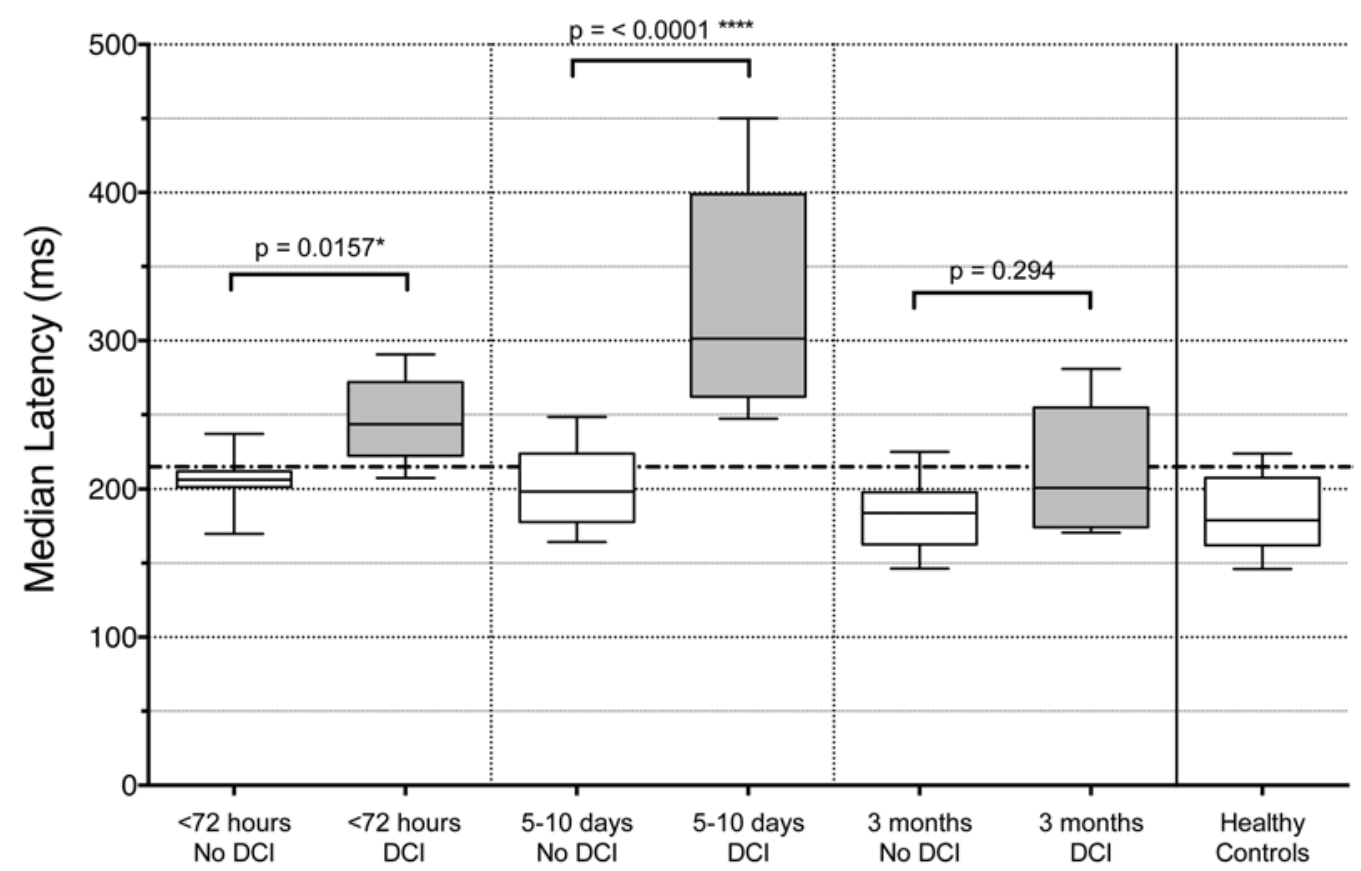

FIG. 3. Differences in median saccadic latency for patients with and without $\mathrm{DCl} / \mathrm{DCl}$-related cerebral infarction in the first 72 hours after aSAH, Days 5-10, and 3 months after aSAH. The dashed and dotted line is placed at 215 msec and represents the median latency cutoff that gives a sensitivity of $88 \%$, a specificity of $65 \%$, and positive predictive value of 0.70 for detecting patients progressing to $\mathrm{DCl}$ and cerebral infarction. ${ }^{*} p<0.05,{ }^{* * * *} p<0.0001$. 
high user variability, require significant training to perform, and have a high false-positive rate (e.g., TCD).

It is probable that the prolonged latencies observed in patients in the first 72 hours following aSAH are a result of acute neuronal damage caused by early brain injury. The control of eye movements is complex and involves a number of different brain areas such as the brainstem, cerebellum, basal ganglia, and cerebral cortex. ${ }^{2}$ Early brain injury occurs in the first 72 hours following aSAH and includes raised intracranial pressure, cerebral edema, microthrombosis, and inflammation, resulting in a global neurological insult. ${ }^{28}$ The nonfocal character of these pathophysiological changes coupled with the heterogeneous functional structure of the oculomotor system makes it difficult to assign the slower saccadic latency to distinct or specific cerebral regions. However, deficits in eye movements are to be expected if the functional integrity of corresponding neural pathways is compromised, as may be the case acutely following aSAH.

Assuming a cutoff value of $215 \mathrm{msec}$ for median saccadic latency in our study population in the first 72 hours after aSAH gives a sensitivity of $88 \%$ for detecting DCI after aSAH, which is a rate comparable to other diagnostic modalities available such as TCD and digital subtraction angiography. Although the specificity was lower at $65 \%$, for a test of DCI this is much more acceptable than a lower sensitivity. Furthermore, compared with other imaging/ clinical approaches to the prediction and diagnosis of DCI, saccadometry is noninvasive and a rapid measurement tool with a very brief learning curve that can be used at the bedside by all levels of clinical staff. The prolonged saccadic latencies in those patients who progress to develop DCI may represent an objective and noninvasive bedside biomarker that might allow earlier and more accurate risk stratification and resource allocation of patients to intensive care interventions (such as invasive pressure monitoring and "triple $\mathrm{H}$ therapy" [induced hypertension, hypervolemia, and hemodilution]) for those patients who might benefit the most.

We studied a cohort that is representative of the standard clinical population of patients with aSAH. There are, however, some potential limitations to the study. The number of subjects recruited is relatively small and further confirmatory studies in larger populations are required. Saccadometry has been shown to have high test-retest reliability over time in healthy volunteers as well as excellent within-test internal consistency. ${ }^{9}$ Currently, this technique cannot be applied to uncooperative patients or those requiring sedation and mechanical ventilation. However, the aim of this study was to target those patients who are stable clinically but at risk for deterioration rather than those who have already been identified as high risk by clinical deterioration. We were also unable to accurately address the question of whether the location of the ruptured aneurysm has an impact on saccadic latency due to the small sample size but hope to investigate this in a future larger study. It was also not possible to obtain data from those patients with cranial nerve palsy, although the incidence of this complication is low, occurring in only approximately $10 \%$ of all WFNS grade patients (i.e., 1-5). ${ }^{17}$ Finally, although all patients had good WFNS grades (Grades 1 or 2) and therefore required no sedative drugs, patients did receive opioid analgesia and other medications such as an- tihypertensives during the study period that were difficult to standardize across recruited participants. This potential effect needs to be investigated in future studies. Regarding other potential confounds such as lack of sleep, sleep deprivation has not been shown to have an effect on reflexive saccades. ${ }^{34}$

\section{Conclusions}

To our knowledge, this is the first study to use saccadometry in patients acutely following aSAH. Our results demonstrate the potential to offer an objective, reliable, and rapid biomarker to predict patients at risk, diagnose DCI early in patients, and facilitate prompt transfer to a critical care unit with the aim of preventing irreversible cerebral damage. Future studies will look to formally validate saccadic latency as a biomarker of DCI and assess whether the addition of saccadometry improves the specificity and sensitivity of current clinical and imaging risk models for the prediction and diagnosis of DCI.

\section{Acknowledgments}

This research was supported by the National Institute for Health Research of Oxford Biomedical Research Centre based at Oxford University Hospitals NHS Trust and the University of Oxford, and by the Medical Research Council (UK). We would like to acknowledge the assistance of Dr. Daniel Lunn, Department of Statistics, University of Oxford, for his statistical analysis in this paper.

\section{References}

1. Antoniades CA, Altham PME, Mason SL, Barker RA, Carpenter R: Saccadometry: a new tool for evaluating presymptomatic Huntington patients. Neuroreport 18:1133-1136, 2007

2. Antoniades CA, Kennard C: Ocular motor abnormalities in neurodegenerative disorders. Eye (Lond) 29:200-207, 2015

3. Antoniades CA, Rebelo P, Kennard C, Aziz TZ, Green AL, FitzGerald JJ: Pallidal deep brain stimulation improves higher control of the oculomotor system in Parkinson's disease. J Neurosci 35:13043-13052, 2015

4. Antoniades CA, Xu Z, Mason SL, Carpenter RHS, Barker RA: Huntington's disease: changes in saccades and handtapping over 3 years. J Neurol 257:1890-1898, 2010

5. Bonnelle V, Leech R, Kinnunen KM, Ham TE, Beckmann $\mathrm{CF}$, De Boissezon X, et al: Default mode network connectivity predicts sustained attention deficits after traumatic brain injury. J Neurosci 31:13442-13451, 2011

6. Cahill J, Calvert JW, Zhang JH: Mechanisms of early brain injury after subarachnoid hemorrhage. J Cereb Blood Flow Metab 26:1341-1353, 2006 (Erratum in J Cereb Blood Flow Metab 26:1463, 2006)

7. Camargo ECS, Furie KL, Singhal AB, Roccatagliata L, Cunnane ME, Halpern EF, et al: Acute brain infarct: detection and delineation with CT angiographic source images versus nonenhanced CT scans. Radiology 244:541-548, 2007

8. Dorsch NWC: Therapeutic approaches to vasospasm in subarachnoid hemorrhage. Curr Opin Crit Care 8:128-133, 2002

9. Ettinger U, Kumari V, Crawford TJ, Davis RE, Sharma T, Corr PJ: Reliability of smooth pursuit, fixation, and saccadic eye movements. Psychophysiology 40:620-628, 2003

10. Frontera JA, Fernandez A, Schmidt JM, Claassen J, Wartenberg KE, Badjatia N, et al: Defining vasospasm after subarachnoid hemorrhage: what is the most clinically relevant definition? Stroke 40:1963-1968, 2009 
11. Heitger MH, Anderson TJ, Jones RD: Saccade sequences as markers for cerebral dysfunction following mild closed head injury. Prog Brain Res 140:433-448, 2002

12. Heitger MH, Jones RD, Macleod AD, Snell DL, Frampton $\mathrm{CM}$, Anderson TJ: Impaired eye movements in post-concussion syndrome indicate suboptimal brain function beyond the influence of depression, malingering or intellectual ability. Brain 132:2850-2870, 2009

13. Hikosaka O, Takikawa Y, Kawagoe R: Role of the basal ganglia in the control of purposive saccadic eye movements. Physiol Rev 80:953-978, 2000

14. Jauch EC, Saver JL, Adams HP Jr, Bruno A, Connors JJB, Demaerschalk BM, et al: Guidelines for the early management of patients with acute ischemic stroke: a guideline for healthcare professionals from the American Heart Association/American Stroke Association. Stroke 44:870-947, 2013

15. Kassell NF, Torner JC, Haley EC Jr, Jane JA, Adams HP, Kongable GL: The International Cooperative Study on the Timing of Aneurysm Surgery. Part 1: Overall management results. J Neurosurg 73:18-36, 1990

16. Kumar G, Shahripour RB, Harrigan MR: Vasospasm on transcranial Doppler is predictive of delayed cerebral ischemia in aneurysmal subarachnoid hemorrhage: a systematic review and meta-analysis. J Neurosurg 124:1257-1264, 2016

17. Laun A, Tonn JC: Cranial nerve lesions following subarachnoid hemorrhage and aneurysm of the circle of Willis. Neurosurg Rev 11:137-141, 1988

18. Leigh RJ, Kennard C: Using saccades as a research tool in the clinical neurosciences. Brain 127:460-477, 2004

19. Lindvall P, Runnerstam M, Birgander R, Koskinen LOD: The Fisher grading correlated to outcome in patients with subarachnoid haemorrhage. Br J Neurosurg 23:188-192, 2009

20. Moppett IK, Mahajan RP: Transcranial Doppler ultrasonography in anaesthesia and intensive care. Br J Anaesth 93:710-724, 2004

21. Müri RM, Nyffeler T: Neurophysiology and neuroanatomy of reflexive and volitional saccades as revealed by lesion studies with neurological patients and transcranial magnetic stimulation (TMS). Brain Cogn 68:284-292, 2008

22. Nicoletto HA, Burkman MH: Transcranial Doppler series part II: performing a transcranial Doppler. Am J Electroneurodiagn Technol 49:14-27, 2009

23. Ober JK, Przedpelska-Ober E, Gryncewicz W, Dylak J, Carpenter R, Ober JJ: Hand-held system for ambulatory measurement of saccadic durations of neurological patients, in Gajda J (ed): Modelling and Measurement in Medicine. Warsaw: PAN, 2003, pp 187-198

24. Pearson BC, Armitage KR, Horner CWM, Carpenter RHS: Saccadometry: the possible application of latency distribution measurement for monitoring concussion. Br J Sports Med 41:610-612, 2007

25. Rowland MJ, Hadjipavlou G, Kelly M, Westbrook J, Pattinson KTS: Delayed cerebral ischaemia after subarachnoid haemorrhage: looking beyond vasospasm. Br J Anaesth 109:315-329, 2012

26. Sandsmark DK, Kumar MA, Park S, Levine JM: Multimodal monitoring in subarachnoid hemorrhage. Stroke 43:14401445,2012

27. Schraa-Tam CKL, van Broekhoven $P$, van der Geest JN, Frens MA, Smits M, van der Lugt A: Cortical and cerebellar activation induced by reflexive and voluntary saccades. Exp Brain Res 192:175-187, 2009

28. Sehba FA, Bederson JB: Mechanisms of acute brain injury after subarachnoid hemorrhage. Neurol Res 28:381-398, 2006

29. Sehba FA, Hou J, Pluta RM, Zhang JH: The importance of early brain injury after subarachnoid hemorrhage. Prog Neurobiol 97:14-37, 2012

30. Straube A, Deubel H, Ditterich J, Eggert T: Cerebellar lesions impair rapid saccade amplitude adaptation. Neurology $\mathbf{5 7 : 2 1 0 5 - 2 1 0 8 , 2 0 0 1}$

31. Topcuoglu MA: Transcranial Doppler ultrasound in neurovascular diseases: diagnostic and therapeutic aspects. J Neurochem 123 (Suppl 2):39-51, 2012

32. Vergouwen MDI, Vermeulen M, van Gijn J, Rinkel GJE, Wijdicks EF, Muizelaar JP, et al: Definition of delayed cerebral ischemia after aneurysmal subarachnoid hemorrhage as an outcome event in clinical trials and observational studies: proposal of a multidisciplinary research group. Stroke 41:2391-2395, 2010

33. Waespe W, Baumgartner R: Enduring dysmetria and impaired gain adaptivity of saccadic eye movements in Wallenberg's lateral medullary syndrome. Brain 115:1123-1146, 1992

34. Zils E, Sprenger A, Heide W, Born J, Gais S: Differential effects of sleep deprivation on saccadic eye movements. Sleep 28:1109-1115, 2005

\section{Disclosures}

The authors report no conflict of interest concerning the materials or methods used in this study or the findings specified in this paper.

\section{Author Contributions}

Conception and design: Rowland, Westbrook, Antoniades, Pattinson. Acquisition of data: Rowland, Garry. Analysis and interpretation of data: Rowland, Corkill, Antoniades. Drafting the article: Rowland. Critically revising the article: all authors. Reviewed submitted version of manuscript: all authors. Approved the final version of the manuscript on behalf of all authors: Rowland. Statistical analysis: Rowland, Antoniades. Administrative/technical/ material support: Rowland. Study supervision: Rowland, Westbrook, Pattinson.

\section{Supplemental Information Online-Only Content}

Supplemental material is available with the online version of the article.

Supplementary Material. https://thejns.org/doi/suppl/10.3171/ 2016.8.JNS16408.

\section{Correspondence}

Matthew Rowland, Nuffield Department of Clinical Neurosciences, University of Oxford, Oxford OX3 9DU, United Kingdom. email: matthew.rowland@ndcn.ox.ac.uk. 